\title{
Influence des conditions de nutrition azotée sur l'accumulation de matière sèche et d'azote par le lupin blanc de printemps
}

\author{
C. Duthion, N. Amarger, \\ avec la collaboration technique de V. Durey, J. Gonthier et P. Mathey \\ INRA, Station d'Agronomie, Laboratoire de Microbiologie des Sols, 17, rue Sully, 21034 Dijon Cedex, France
}

(reçu le 12-1-1988, accepté le 12-10-1988)

Résumé - En vue d'apprécier l'influence des conditions de nutrition azotée sur le comportement du lupin blanc, des essais factoriels avec les deux variables inoculation et fertilisation azotée au semis ont été mis en place en sols pauvres en Rhizobium lupini. Dans un essai complémentaire on a fait varier la date d'apport de l'engrais.

La fixation symbiotique a permis croissance pondérale, mobilisation en $\mathrm{N}$, rendements maximaux. L'apport de $\mathrm{N}$ au semis aux plantes inoculées (I) a seulement augmenté leur croissance végétative. Les résultats obtenus avec les plantes non inoculées $(\mathrm{NI})$ et non fertilisées ont été très inférieurs. L'application aux plantes NI de $160 \mathrm{~kg} \mathrm{~N} / \mathrm{ha}$ au semis a entraîné sensiblement les mêmes accumulation de MS et rendement en graines que sur parcelles I mais les teneurs $\mathrm{N}$ de la plante entière et des graines sont demeurées faibles. Des doses d'engrais plus élevées ne les ont pas augmentées.

Lorsque les 2 voies de la nutrition azotée ont été accessibles à la plante, elles ont été plutôt complémentaires qu'antagonistes; la plante a absorbé $\mathrm{N}$ minéral selon sa disponibilité dans le sol et couvert ses besoins par un complément d'origine symbiotique. Toute la phase d'élaboration du nombre de graines récoltables est une phase sensible vis-à-vis de la nutrition azotée.

\section{fixation symbiotique - assimilation $\mathbf{N}$ - répartition MS - répartition $\mathbf{N}$ - phase sensible}

Summary - Influence of nitrogenous nutrition conditions on dry matter and nitrogen accumulation in the spring white lupin. Factorial experiments were set up on soils with a low Rhizobium lupini content, to assess the influence of nitrogenous nutrition conditions on spring white lupin behaviour; the two variables were inoculation, and mineral nitrogen provided at sowing. A supplementary experiment was carried out, in which the date of fertiliser deposit was altered.

Symbiotic fixation resulted in maximal weight growth, $N$ accumulation and yield. When inoculated plants (I) received $N$ at sowing, only their vegetative growth increased. The results obtained with non inoculated (NI) and unfertilised plants were much lower than with inoculated plants. When non inoculated plants received $160 \mathrm{~kg} \mathrm{~N} / \mathrm{ha}$ at sowing, DM accumulation and seed yields were practically the same as those for inoculated plots; but $N$ content of plants and seeds remained low. Increased amounts of fertiliser did not have an effect.

When the plants were able to utilise the two means of $N$ nutrition, they proved to be complementary rather than antagonistic; plants absorbed mineral $N$ according to its availability in the soil, and provided for additional needs with a symbiotic complement. The stage during which the number of viable seeds is formed is greatly affected by $N$ nutrition.

\section{$N$ symbiotic fixation $-\boldsymbol{N}$ assimilation $-D M$ distribution $-\boldsymbol{N}$ distribution $-\boldsymbol{N}$ sensitive phase}

\section{Introduction}

Dans un article précédent (Duthion et al., 1987), nous avons décrit l'évolution de l'accumulation de matière sèche (MS) et d'azote par le lupin blanc (Lupinus albus L.) de printemps au cours de son cycle. Les informations avaient été obtenues en conditions de nutrition azotée non limitantes; la fixation symbiotique, efficiente, avait assuré $80 \%$ de l'accumulation de $\mathrm{N}$. Les résultats culturaux correspondants, se situant au niveau des meilleurs rendements et teneurs en $\mathrm{N}$ des récoltes qui 
aient été observés, nous permettaient de présenter ces données comme des références possibles pour l'espèce.

Selon les situations culturales, les modalités d'alimentation azotée d'une légumineuse à graines diffèrent; les interactions entre les 2 voies d'absorption de $\mathrm{N}$, fixation symbiotique et assimilation de $\mathrm{N}$ minéral, et l'action différenciée des facteurs du milieu sur l'une et l'autre en sont causes.

L'inhibition de la fixation symbiotique par $N$ minéral présent dans le sol ou introduit a été maintes fois rapportée mais l'influence bénéfique d'une fumure $\mathrm{N}$ en début de végétation est quelquefois mise en évidence (Merbach et al., 1980); la quantité disponible, donc la fertilité du sol ou la place de la légumineuse dans la rotation, la date et l'importance de l'apport déterminent le sens de l'effet. Un exemple d'inhibition de l'assimilation par la fixation a été relevé chez le lupin blanc (Montfort, 1978).

Certains des facteurs du milieu paraissent agir davantage sur la fixation symbiotique que sur l'assimilation de $\mathrm{N}$ minéral; leur influence est souvent indirecte. Tel est le cas notamment du régime hydrique, qui est fréquemment invoqué (Obaton et al., 1982). Dans quelques situations enfin, la fixation s'établit difficilement, parce que le nombre de bactéries de l'espèce inféodée à la plante est insuffisant, ou est peu efficiente en raison de la souche de Rhizobium présente ou de l'association plante-Rhizobium réalisée.

Lors d'essais mis en place dans un sol pauvre en $R$. lupini, nous avons conduit des parcelles de lupin blanc en conditions différenciées de nutrition azotée, au moyen des 2 traitements inoculation et/ou fertilisation minérale (Amarger et Duthion, 1983). Les données que nous avons proposées comme références proviennent des parcelles inoculées mais non fertilisées incluses dans ces essais. Par la comparaison des différentes situations créées, nous tentons d'apporter des informations concernant l'influence du mode de nutrition azotée sur les accumulations de MS et de $\mathrm{N}$, du niveau de celles-ci sur les répartitions et redistributions, l'effet inhibiteur de $\mathrm{N}$ minéral sur la fixation symbiotique chez le lupin blanc.

Pour une certaine clarté de la présentation, seuls seront exposés les résultats de l'une des trois années d'expérimentation, correspondant aux situations réalisées les plus contrastées; les résultats des deux autres années n'infirment aucune des conclusions qui pourront en être tirées. Les rendements obtenus dans un essai complémentaire faisant varier doses et dates d'apport de $\mathrm{N}$ minéral seront également donnés.

\section{Matériel et Méthodes}

\section{Essais 1979, 1980 et 1981}

Les dispositifs expérimentaux mis en place en 1979 , 1980 et 1981 ont été précédemment décrits (Duthion et al., 1987). Les 2 facteurs de variation introduits ont été l'inoculation par $R$. lupini (oui/non) et la fertilisation azotée au semis $(0,40,80$ ou $160 \mathrm{~kg} \mathrm{~N} /$ ha sous forme d'ammonitrate). Les résultats détaillés ici sont ceux qui ont été obtenus en 1981, complétés par les données de l'essai 1983, présenté ci-dessous. Au cours de ces 2 années, les nodulations dues à des $R$. lupini indigènes ou consécutives à de possibles contaminations ont été rares et tardives, interférant peu avec les traitements appliqués. Les différents traitements sont symbolisés dans le texte par I (inoculation) et NI (sans inoculation) suivi du nombre correspondant à la quantité de $\mathbf{N}$ apportée.

Le cultivar (cv) Kali a été utilisé. L'article cité en référence contient les données relatives aux caractéristiques du sol, conditions climatiques, bilan hydrique et conduite de la culture; les prélèvements de plantes, observations, mesures et analyses effectués y sont également indiqués.

Estimé en tenant compte de la texture du sol, de la pluviométrie et de l'évapo-transpiration, le front de migration de $\mathrm{N}$ de l'engrais un mois après l'apport s'est situé à environ $30 \mathrm{~cm}$ de profondeur. L'évolution de la quantité de $\mathrm{N}$ minéral dans le sol, sous parcelles de différents traitements est représentée dans la Figure 1. Les quantités d'azote disponibles se situent sensiblement au même niveau sur toutes les parcelles, fertilisées ou non, dès la mi-juin, au moment de la floraison A3.

\section{Essai 1983}

En 1983, un essai complémentaire a été mis en place sur le même type de sol qu e les essais précédents. Le cv Kali a été semé le 11 mars; la levée a eu lieu le 5 avril, le début de la floraison le 1er juin et la récolte le 9 août. Le peuplement réalisé a été de 74 plantes $/ \mathrm{m}^{2}$. La saison a été caractérisée par les très fortes précipitations des mois d'avril et mai. Un arrosage de $50 \mathrm{~mm}$ a été fait fin juin; le déficit hydrique cumulé (ETM - P) a dépassé la valeur de la réserve utile du sol seulement dans les trois dernières semaines du cycle.

Etabli selon un dispositif factoriel en blocs avec 4 répétitions, l'essai portait sur les 3 variables inoculation (oui/non), fertilisation azotée $(0,80,160,240$ ou $320 \mathrm{~kg}$ $\mathrm{N} / \mathrm{ha}$ ), modalité d'apport (apport en 1 fois au semis, ou fractionné par moitiés au semis et à la mi-floraison). Les 2 apports prévus ont été faits, sous forme d'ammonitrate, le 14 mars et le 15 juin. Le niveau moyen de l'onde de migration de l'azote épandu à la $1^{\text {re }}$ date devant se situer vers $70 \mathrm{~cm}$ de profondeur à la fin de la période des fortes pluies, une fraction de cet azote, estimée à $50 \%$, a été entraînée au-delà de la zone explorée par les racines. Un apport compensateur, égal à cette fraction, a été effectué le 27 mai. La migration de l'azote épandu à cette date et le 15 juin n'a pas dépassé la profondeur de la couche labourée. Aucun contrôle en cours 


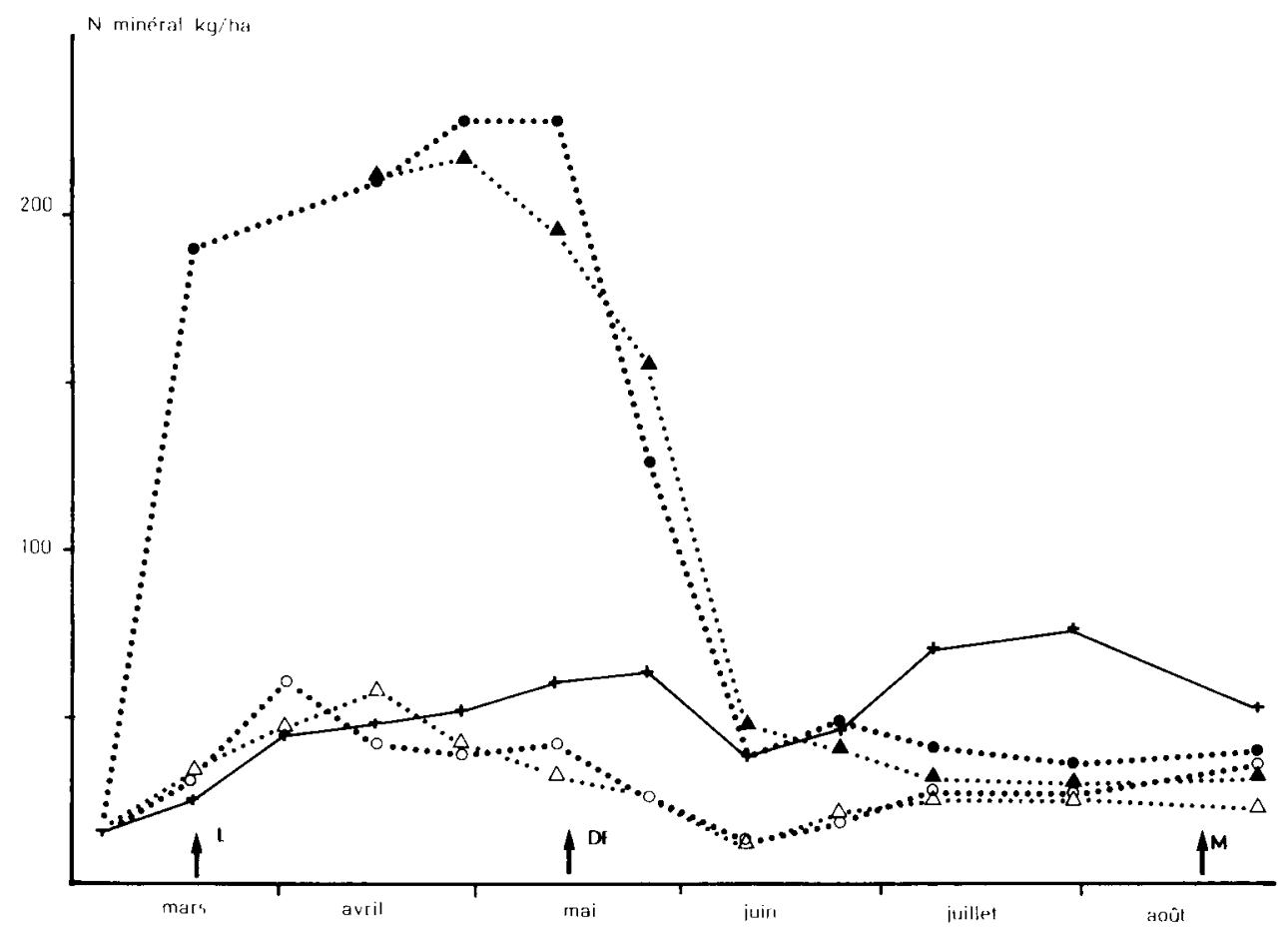

Fig. 1. Evolution de $\mathrm{N}$ minéral dans le sol $(0-40 \mathrm{~cm}) .+$ sol nu, $O$ parcelles inoculées $O \mathrm{~N}$, - parcelles inoculées $160 \mathrm{~N}, \angle$ parcelles non inoculées $0 \mathrm{~N}, \Delta$ parcelles non inoculées $160 \mathrm{~N}, \mathrm{~L}=$ levée, $\mathrm{DF}=$ début floraison, $M=$ maturité.

de végétation n'a été réalisé. Le rendement a été mesuré par récolte mécanique. La détermination de la teneur $\mathrm{N}$ des graines a été faite selon Kjeldahl.

\section{Résultats}

\section{Déroulement du cycle végétatif}

Les dates des événements retenus comme repères dans le cycle (Duthion et al., 1987) ont été semblables dans toutes les conditions de nutrition azotée. En l'absence d'émission des axes $\mathrm{A3}^{*}$ et de fructification $\mathrm{A} 2$, la maturité des plantes NIO a cependant été un peu plus précoce que pour les autres traitements, le décalage par rapport à ces derniers étant inférieur à 1 semaine (environ $100^{\circ} \mathrm{C} . \mathrm{j}$ ).

Production de matière sèche aérienne et accumulation d'azote

Influence d'un apport de $N$ au semis sur parcelles inoculées

L'apport de $\mathrm{N}$ minéral au semis n'a pas modifié la durée des différentes phases d'accumulation de la

\footnotetext{
* L'axe principal de la plante est symbolisé par $\mathrm{AO}$, les axes apicaux d'ordre 1 par A1, les axes apicaux d'ordre 2 par A2 et ceux d'ordre 3 par A3.
}

MS (Fig. 2; dans cette figure et ce qui suit, le temps a été exprimé en somme de températures base $0^{\circ} \mathrm{C}$, pour maintenir la comparaison avec Duthion et al., 1987).

Les valeurs maximales de la MS totale élaborée par 10 et 1160 n'ont pas été différentes. En début de cycle, pourtant, l'accumulation a été plus forte chez les plantes 1160; le poids de leur appareil végétatif était alors significativement plus élevé et l'est demeuré jusqu'à ce que la progression de la chute des feuilles ait touché les axes $A 1$ (vers $1400^{\circ} \mathrm{C}$.j). Les augmentations de MS totale et végétative notées dans les 1 ers stades après fertilisation par $80 \mathrm{~N}$ n'ont été qu'irrégulièrement significatives; le traitement $40 \mathrm{~N}$ n'a pas eu d'effet. Le poids de l'appareil reproducteur n'a été modifié par aucune des doses d'engrais.

Après les apports de 80 et $160 \mathrm{~N}$, la quantité de $\mathrm{N}$ accumulé a été significativement plus forte dès le début de cycle, au profit de l'appareil végétatif, et elle l'est restée jusque vers $1300^{\circ} \mathrm{C}$.j; mais ces apports n'ont pas accru la valeur maximale de $\mathrm{N}$ accumulé, ni le contenu en $\mathrm{N}$ de l'appareil reproducteur. La dose inférieure n'a pas eu d'effet significatif (Fig. 3).

La fertilisation n'a pas retardé la date à laquelle a débuté l'activité fixatrice d'azote des plantes ni abrégé la période pendant laquelle elle s'est exercée. Elle a en revanche fortement influé sur 


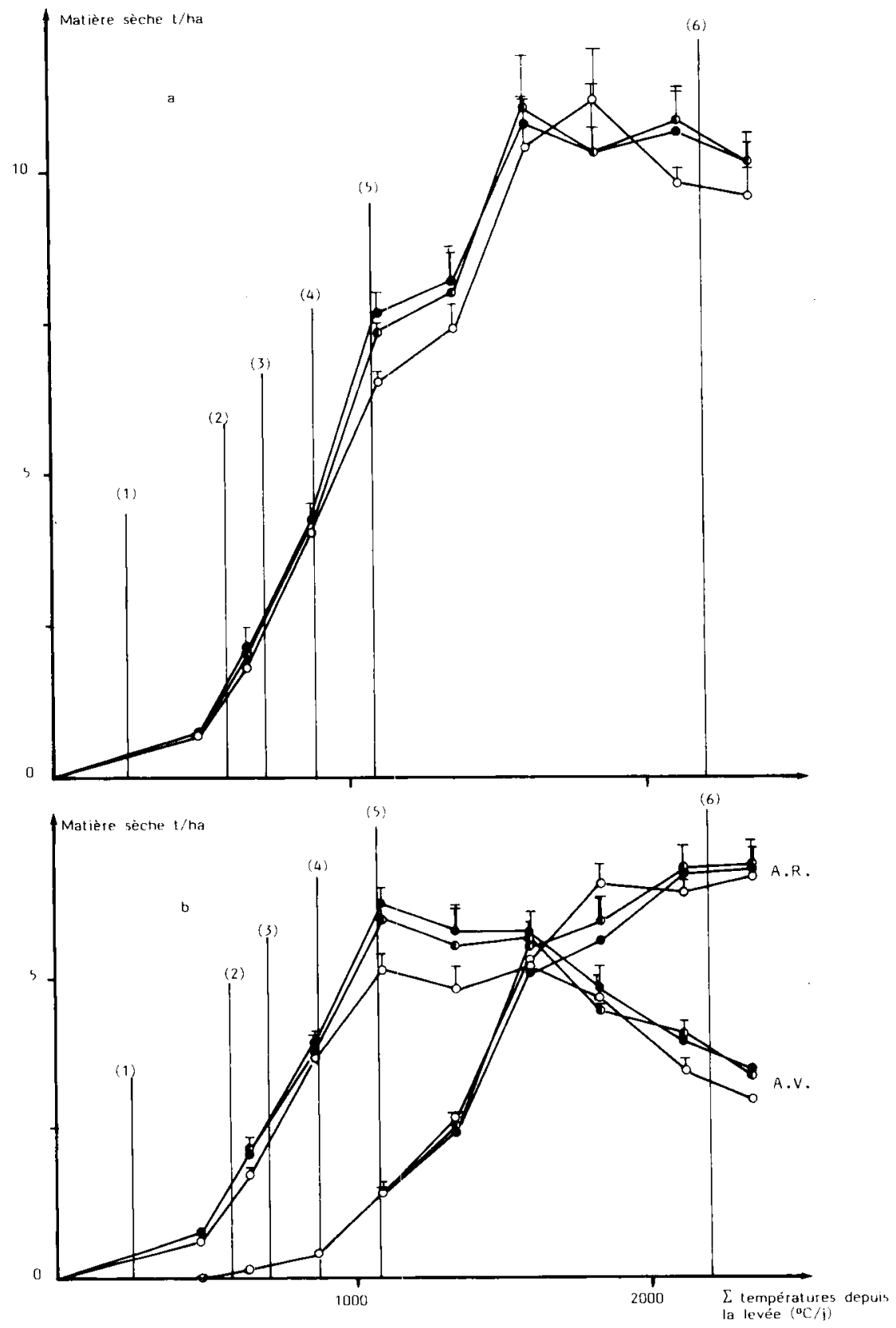

Fig. 2. Production de MS aérienne tha (Fig. 2a) et répartition entre appareils végétatif et reproducteur (Fig. 2b). Les demi-droites verticales indiquent quelques stades repères : (1) 5 feuilles ou début allongement tige, (2) floraison $A 0$, (3) floraison $A 1$, (4) floraison $A 2$, (5) floraison A3, (6) maturité. Les segments verticaux représentent les écarts types des moyennes. $O$ parcelles inoculées $0 \mathrm{~N}, \mathbf{0}$ parcelles inoculées $80 \mathrm{~N}, \bullet$ parcelles inoculées $160 \mathrm{~N}$, A.R. = appareil reproducteur et cosses + graines, A.V. = appareil végétatif tiges + feuilles.

son niveau (Fig. 4); le $\mathrm{N}$ total au moment de la floraison $\mathrm{A} 3$ et à la récolte (dates 2 et 3 de la Figure 4) n'est d'origine symbiotique respectivement que pour 38 et $50 \%$ sur les parcelles 1160 au lieu de $80 \%$ sur les parcelles 10 .

La part du $\mathrm{N}$ contenu dans les graines à la récolte qui proviendrait de redistribution à partir des organes végétatifs et des cosses des gousses et celle qui viendrait du $\mathbf{N}$ incorporé par la plante au cours de la phase de remplissage des graines ont été évaluées de la façon précédemment décrite (Duthion et al., 1987). L'apport d'azote ne les a pas modifiées significativement (Fig. 5). La proportion de $\mathrm{N}$ fixé est significativement inférieure pour les parcelles fortement fertilisées dans $N$ venant des redistributions; elle est du même ordre de grandeur pour tous les traitements dans $\mathbf{N}$ incorporé en fin de cycle. 


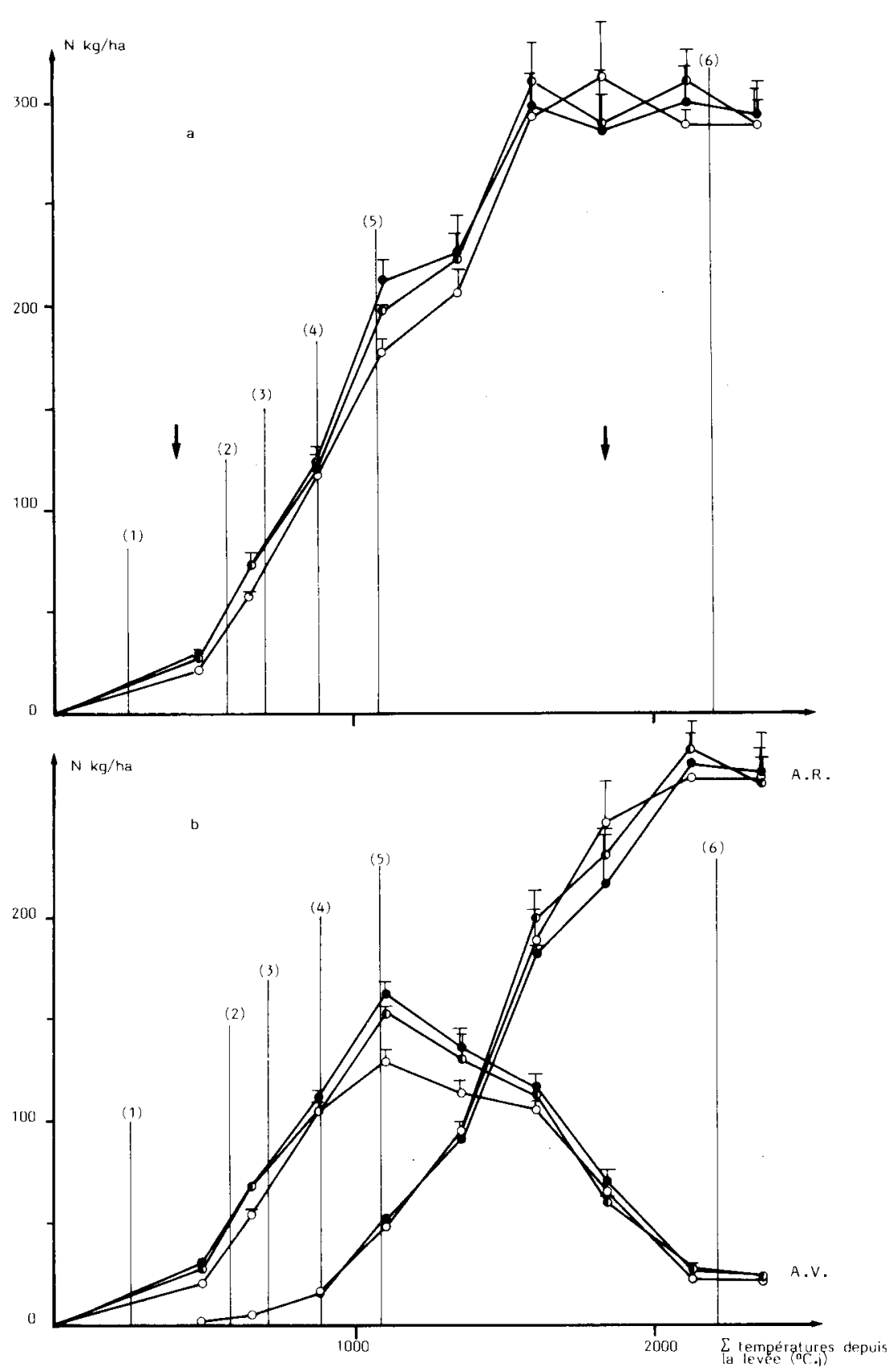

Fig. 3. Accumulation d'azote dans les parties aériennes kg/ha (Fig. 3a) et répartition entre organes végétatifs et reproducteurs (Fig. $3 b)$. \ début et fin d'activité fixatrice. Pour la signification des autres repères et des symboles, voir la Figure 2. Les segments verticaux représentent les écarts types des moyennes.

Accumulations en situation de fixation faible ou tardive

Une telle situation a été réalisée en l'absence d'inoculation. Des nodulations tardives ont été notées; elles se sont manifestées, entre 1350 et $1600^{\circ} \mathrm{C} . \mathrm{j}$, par un reverdissement progressif de plantes, en nombre et position aléatoires à l'intérieur des parcelles.
L'accumulation de MS par les plantes NIO a été inférieure à celle des plantes 10 dès le début floraison; sa valeur maximale a été d'environ 7 tha, atteinte vers $1600^{\circ} \mathrm{C}$.j (Fig. 6). Le poids de l'appareil végétatif et le poids de l'appareil reproducteur ont été réduits dans des proportions peu différentes par rapport à ceux de 10 .

La fertilisation a augmenté la croissance pondérale des plantes NI (Fig. 6). Le niveau d'accumula- 


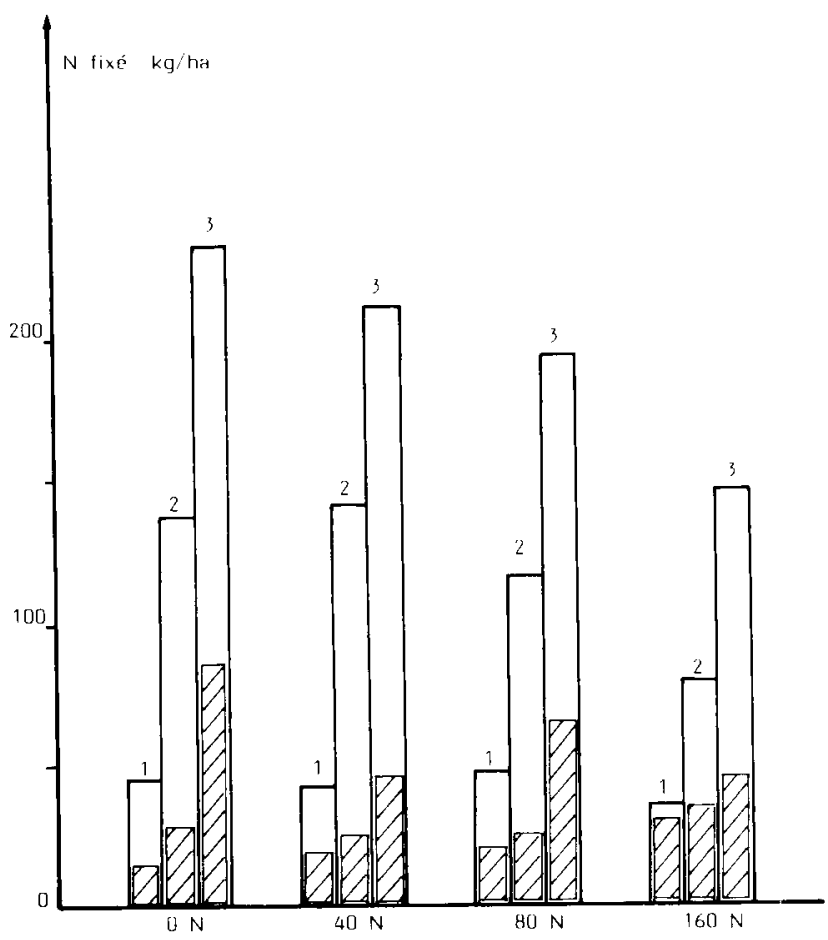

Fig. 4. Quantités d'azote fixé, en fonction des différents apports de $\mathrm{N}$ minéral, à 3 dates : 1 floraison $\mathrm{A} 0,2$ floraison $\mathrm{A} 3$, 3 récolte. Rectangles vides: parcelles inoculées; rectangles hachurés : parcelles non inoculées.

tion de MS totale, dépendant de la dose apportée, a été identique à celui de 10 pour $160 \mathrm{~N}$. En début de cycle, la croissance végétative a été plus importante sur N180 et 160 que sur 10. Le poids de l'appareil reproducteur a été d'autant plus proche de celui de 10 que la dose d'engrais a été plus élevée. Pour tous ces traitements, les phases d'accumulation ont été bornées aux mêmes dates que pour 10.

La réduction, relativement à 10 , de l'accumulation de $\mathrm{N}$ a été antérieure à celle de la production de MS (Fig. 7). Les valeurs maximales de $\mathrm{N}$ total ont varié de 150 à $200 \mathrm{~kg} / \mathrm{ha}$ selon le niveau de fertilisation. L'accumulation de $\mathrm{N}$ par les plantes fortement fertilisées a eu lieu pour sa plus grande part avant fin floraison; en revanche elle s'est faite pour plus de moitié après cette date sur les parcelles NIO. Par rapport aux valeurs correspondantes de 10 , les quantités de $\mathrm{N}$ végétatif ont été beaucoup plus faibles pour les plantes NIO, passagèrement supérieures dans les 1 ers stades pour les plantes fertilisées. L'accumulation de $\mathrm{N}$ dans les organes reproducteurs a été inférieure pour tous les NI, mais dépendante de l'apport d'engrais.

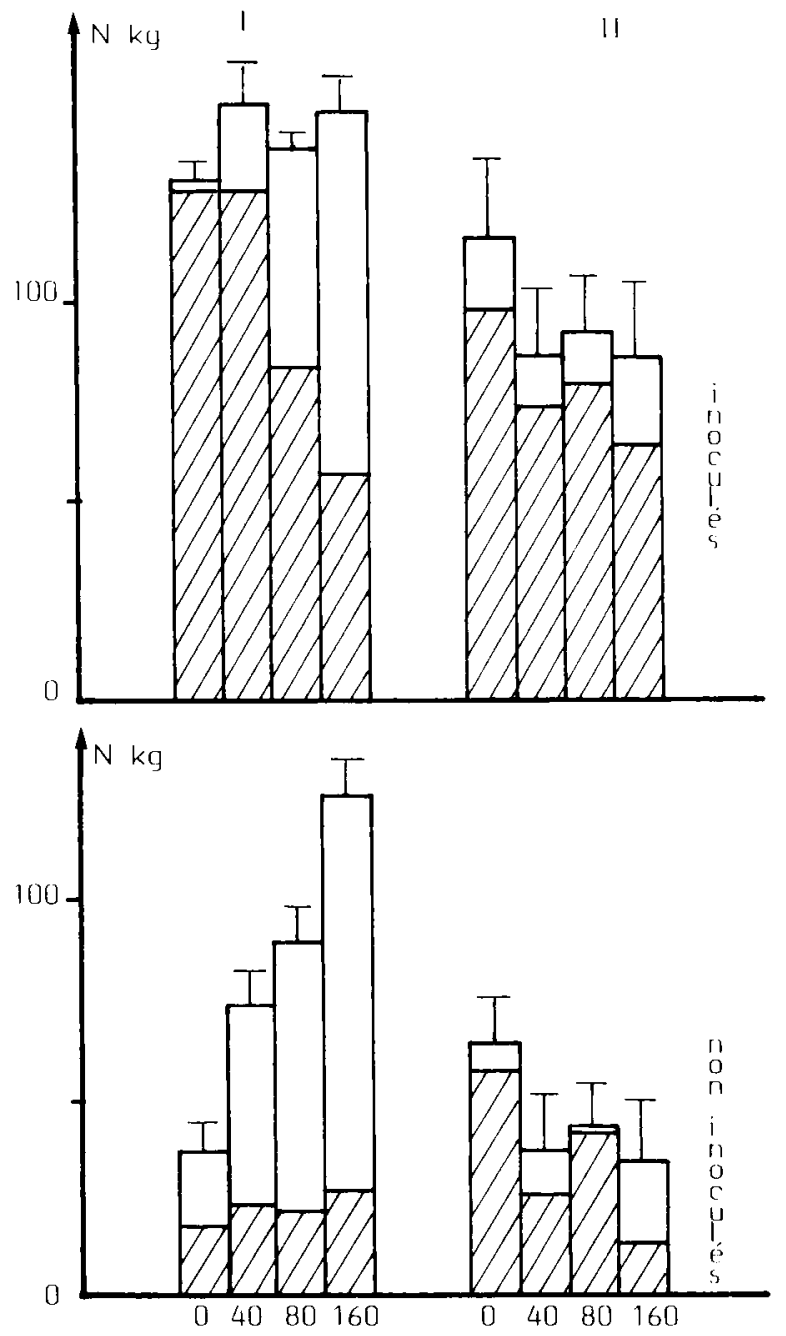

Fig. 5. Provenance de l'azote des graines. 1 : redistributions à partir des organes végétatifs et cosses; II : accumulation pendant la phase de remplissage. En hachuré : azote d'origine symbiotique. Les segments verticaux représentent les écarts types des moyennes.

L'activité fixatrice des plantes $\mathrm{NI}$ a été faible par rapport à celle des plantes I (Fig. 4). Cependant elle a assuré, particulièrement dans la phase terminale du cycle, une grande part des accumulations de $\mathrm{N}$ de certains traitements; à maturité, $70 \%$ du contenu en N de NIO est d'origine symbiotique, mais seulement $25 \%$ de celui de NI 160 .

L'azote des graines des plantes NI160 provient pour les $3 / 4$ de redistributions à partir des organes végétatifs et cosses (Fig. 5); ces quantités redistribuées sont du même ordre de grandeur que pour les plantes I. Sans fertilisation, le N des graines vient pour $2 / 3$ de $\mathrm{N}$ incorporé pendant la phase de remplissage. 

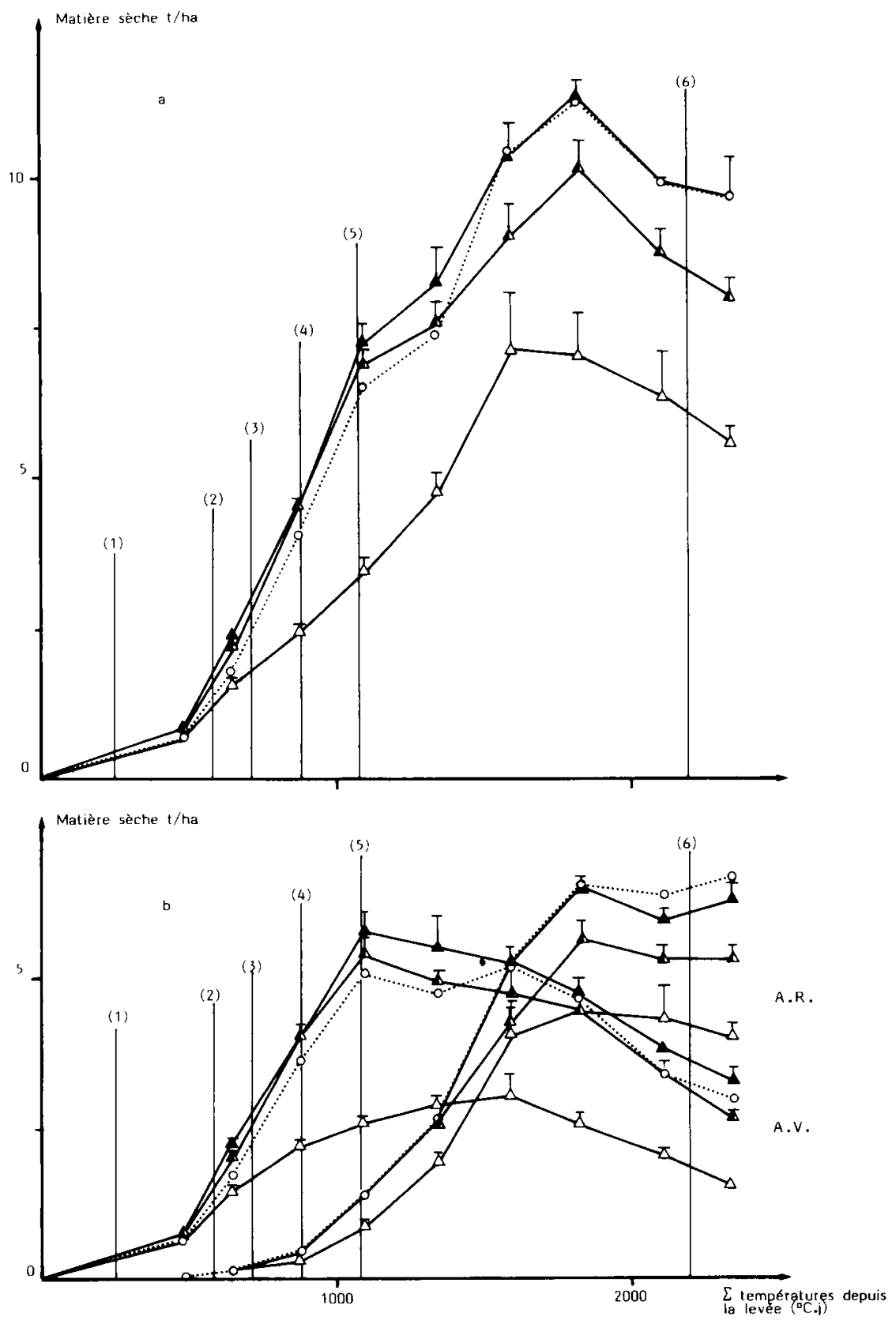

Fig. 6. Production de MS aérienne tha (Fig. 6a) et répartition entre appareils végétatif et reproducteur (Fig. 6b). $O$ parcelles inoculées $0 \mathrm{~N}, \Delta$ parcelles non inoculées $0 \mathrm{~N}, \Delta$ parcelles non inoculées $80 \mathrm{~N}, \Delta$ parcelles non inoculées $160 \mathrm{~N}$. Pour la signification des repères et autres symboles, voir la Figure 2 . Les segments verticaux représentent les écarts types des moyennes.

\section{Résultats culturaux}

\section{Essai 1981}

Les rendements machine, les teneurs des graines en $\mathrm{N}$ et matières grasses sont rappelés dans la Figure 8. L'effet de l'inoculation sur les rendements a été hautement significatif. La fertilisation des parcelles I a entraîné une légère diminution de leur production, significative pour $160 \mathrm{~kg} / \mathrm{ha}$; elle a accru en revanche celle des parcelles NI, la portant, à la forte dose, au même niveau que sur parcelles I. Les différences de rendement sont imputables pour l'essentiel aux nombres de graines produites et non au poids de ces graines (Fig. 8). L'examen de la production par ordre d'axes a montré qu'inoculation et fertilisation tendaient à accroître la part venant des axes apicaux. Les teneurs $\mathrm{N}$ des récoltes ont été très supérieures après inoculation, mais n'ont pas été influencées 

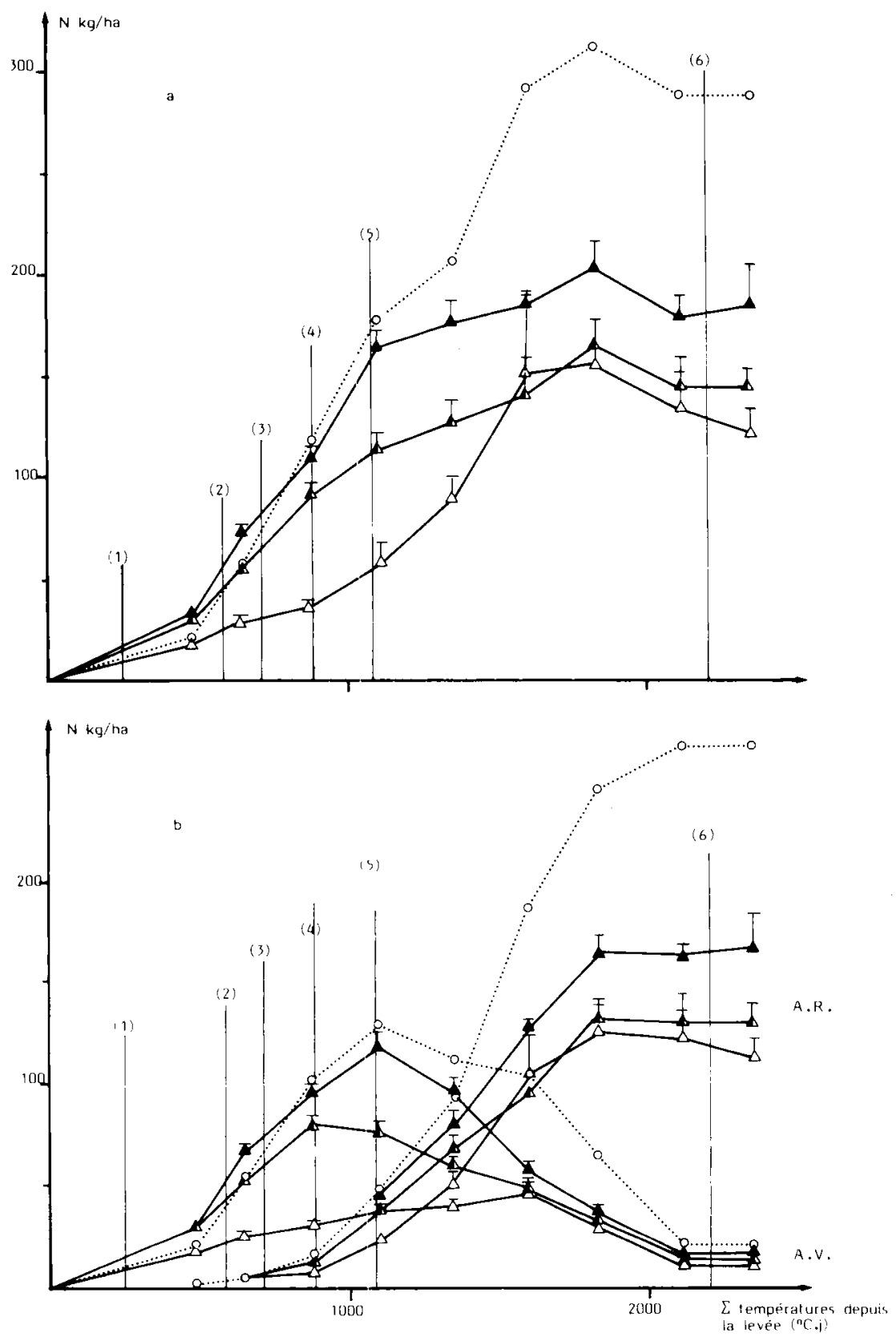

Fig. 7. Accumulation d'azote dans les parties aériennes $\mathrm{kg} /$ ha (Fig. $7 \mathrm{a}$ ) et répartition entre appareils végétatif et reproducteur (Fig. $7 \mathrm{~b}$ ). Pour la signification des repères et des symboles, voir les Figures 2 et 6 . Les segments verticaux représentent les écarts types des moyennes.

par la fertilisation; les teneurs en matières grasses sont plus élevées pour les graines pauvres en $\mathrm{N}$.

Les indices de récolte pour la MS, calculés à partir des échantillons de plantes prélevés à maturité, ont été significativement différents sur parcelles I et sur parcelles $\mathrm{NI}$, ayant pour valeurs moyennes 0,44 et 0,37 (les productions estimées sur la base de ces prélèvements sont supérieures de 8 et $6 \%$ aux récoltes machine respectivement pour 10 et NIO); en l'absence d'inoculation, l'apport de $\mathrm{N}$ les a réduits significativement (Fig. 8). L'indice de récolte pour $N$, légèrement supérieur à 0,8 , n'a pas été influencé par les traitements.

\section{Essai 1983}

L'analyse des rendements fait apparaître, comme dans l'essai précédent, un effet inoculation haute- 

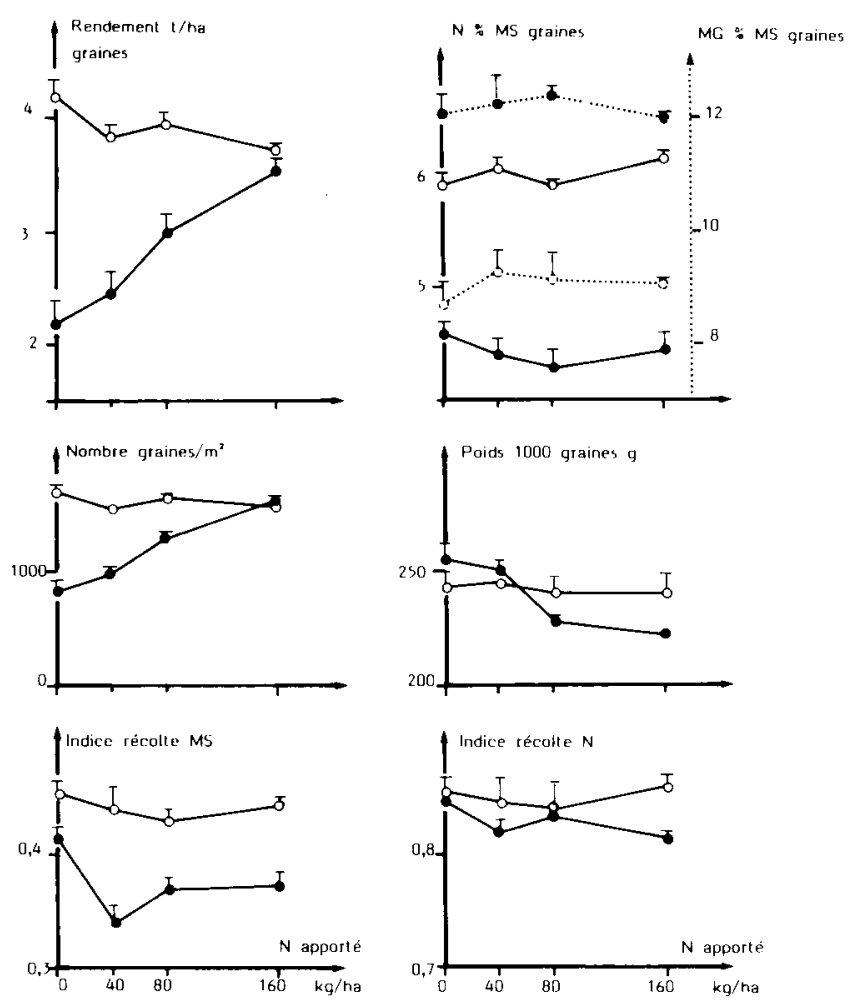

Fig. 8. Résultats culturaux obtenus sur l'essai 1981. O parcelles inoculées, - parcelles non inoculées; les segments verticaux représentent les écarts types des moyennes.

ment significatif. Aucun des apports d'engrais n'a modifié la production des parcelles I (Fig. 9). la fertilisation a entraîné en revanche une augmentation de celles des parcelles NI; avec une application unique au semis le rendement a augmenté jusqu'à la dose $160 \mathrm{~kg} / \mathrm{ha}$, avec un apport fractionné l'accroissement a été moindre mais encore significatif entre 160 et $320 \mathrm{~kg} / \mathrm{ha}$.

Les teneurs en $\mathrm{N}$ des graines ont été nettement différentes avec et sans inoculation mais n'ont pas varié après fertilisation, quel qu'en ait été le mode d'application.

\section{Discussion et Conclusion}

\section{Comportement en fonction des conditions de nutrition azotée}

La fertilisation $N$, appliquée peu après le semis ou fractionnée avec un apport à mi-floraison, a globalement peu modifié le comportement des plantes I. Le facteur $\mathrm{N}$ a été manifestement limitant en l'ab-

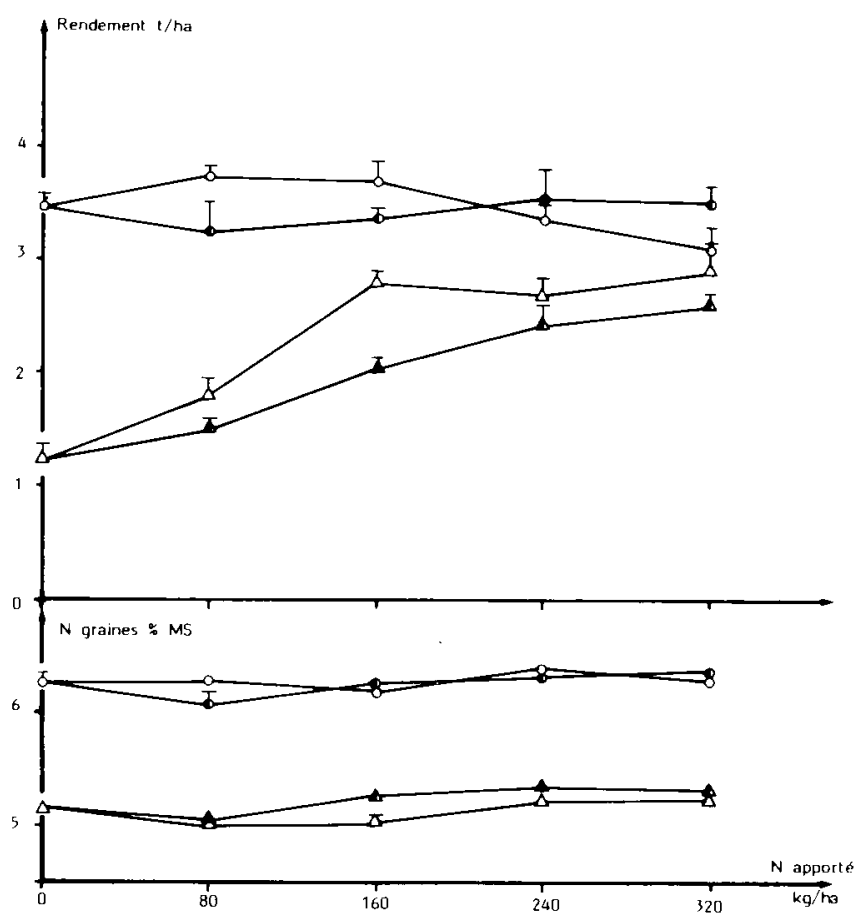

Fig. 9. Résultats culturaux obtenus sur l'essai 1983. Parcelles inoculées : $\mathrm{O}$ apporté au semis, $\mathbf{N}$ apporté $1 / 2$ au semis, $1 / 2$ pendant la floraison. Parcelles non inoculées : $\Delta N$ apporté au semis, $\Delta N$ apporté $1 / 2$ au semis, 1/2 pendant la floraison. Les segments verticaux représentent les écarts types des moyennes.

sence d'inoculation (Figs. 6 et 7), et dans cette situation, en revanche, l'apport d'engrais a entraîné une augmentation de la plupart des grandeurs mesurées; elles n'ont cependant pas toutes atteint le même niveau que sur parcelles I, même avec les plus fortes doses épandues.

Les résultats présentés sont en accord avec les observations faites sur d'autres espèces de légumineuses à graines, en particulier le soja. Les nombreux essais de fertilisation azotée du soja ont montré son inefficience dans la plupart des cas et, lorsqu'il y a augmentation de rendement, elle est faible sauf dans quelques essais de fertilisation tardive (Bouniols et al., 1986). Comparant des lignées non nodulantes de soja à leurs lignées isogéniques nodulantes, Hanway et Weber (1971 a; 1971 b), Pal et Saxena (1976), Weber (1966 a) ont mis en évidence l'absence d'effet de la fertilisation sur le rendement et la teneur des plantes fixatrices, l'augmentation de la MS totale, de N accumulé, du rendement et parfois de la teneur des graines des plantes non fixatrices après application de $\mathrm{N}$ minéral; les quantités d'engrais nécessaires pour porter les performances de ces 
plantes au niveau de celles des lignées nodulantes sont élevées et dépendent des conditions de croissance.

L'accumulation de $\mathrm{N}$ a été parallèle à celle de la MS chez les plantes I mais non chez les plantes NI (Figs. 2-3 et 6-7). Au cours du cycle, la teneur $\mathrm{N}$ moyenne de ces dernières a diminué jusqu'à une valeur minimale, puis augmenté légèrement sans doute en raison d'une efficience tardive accrue de leur activité fixatrice. Cette valeur minimale, atteinte à la floraison A2 par NI0, plus tardivement par N140, a été un peu inférieure à 1,5\% de la MS pour ces 2 traitements. Une telle teneur est voisine de la concentration minimale chez les légumineuses, au-dessous de laquelle cesserait, selon Penning de Vries (1982), le fonctionnement de la plante; or elle n'a pas entraîné d'arrêt apparent de la croissance pondérale (Fig. 6). Le niveau moyen dans la plante entière dissimule des états très différents, en particulier parmi les organes de la photosynthèse; pour les plantes NIO et 40 , au moment où leur teneur $\mathrm{N}$ moyenne était minimale, celle des feuilles AO était d'environ $2 \%$, mais celle des feuilles des axes les plus élevés de 3 à $3,5 \%$, nettement supérieure au taux de 1,6\% mesuré dans leurs feuilles après sénescence. En comparaison, la teneur $\mathrm{N}$ des plantes I s'est établie au niveau constant de 2,8 à $3 \%$ après élaboration des 2 premières tonnes de MS; elle a varié de 5 à $6 \%$ dans les feuilles les plus jeunes à 2 à $2,5 \%$ dans les feuilles sénescentes.

Les différences de rendement entre traitements sont imputables pour l'essentiel à la composarite nombre de graines produites par unité de surface. La limitation de $\mathrm{N}$ pendant la période, débutant à la floraison, au cours de laquelle se poursuit la mise en place des structures de la plante et s'élabore le nombre de graines, a eu un effet dépressif sur celui-ci; plus la limitation a été précoce, plus l'effet a été marqué (traitements NIO, 40 et 80 ). L'ensemble de la période peut donc être considérée comme une phase sensible; chez le soja, Brevedan et al. (1978) ont souligné aussi les besoins élevés en $\mathrm{N}$ pendant la floraison et la nouaison pour assurer un nombre maximal de graines. La réduction plus tardive de l'accumulation de $\mathrm{N}$ pour $\mathrm{Nl} 160$ a permis d'élaborer un nombre de graines semblable à celui de I160 mais l'absence d'ajustement aux capacités ultérieures de remplissage a entraîné une diminution du poids de la graine. Toute déficience avant la fin de franchissement du stade limite d'avortement des graines provoque donc une réduction de leur nombre, au-delà, elle limite leur remplissage.

Le nombre de graines récoltables ne paraît pouvoir dépasser 1600 à 1700 graines élaborées par $\mathrm{m}^{2}$ pour les $\mathrm{cv}$ actuels de lupin blanc.

Les résultats obtenus avec les plantes NI démontrent une grande plasticité de l'espèce pour la teneur en $\mathrm{N}$ de ses graines, les valeurs mesurées dans ce cas étant de beaucoup inférieures à toutes celles qui ont été répertoriées par Hill (1977).

\section{Répartition de la MS et de N}

Dans tous les cas, MS et $\mathbf{N}$ végétatifs ont été accumulés pour leur plus grande part au cours de la floraison, atteignant leurs valeurs maximales à la fin de cette période. A ce moment, la répartition des assimilats $\mathrm{C}$ et $\mathrm{N}$ traduit la compétition qui s'est exercée entre les appareils végétatif et reproducteur. Dans les limites des quantités observées à cette date, le pourcentage de la MS et de N investis dans chacun d'eux a été directement dépendant de leur niveau d'accumulation dans la plante entière; l'inoculation et/ou la fertilisation ont provoqué une augmentation du pourcentage attribué aux organes végétatifs, sensiblement proportionnelle à l'accroissement total. La répartition de la MS végétative entre tiges et feuilles a été peu modifiée par les traitements. En revanche, le rapport $\mathrm{N}$ tiges/ $\mathrm{N}$ feuilles (non présenté dans les résultats) a été pour $\mathrm{NI} 0$ et 40 très inférieur à celui de 10, la répartition favorisant dans ce cas l'activité photosynthétique de la plante.

L'indice de récolte pour la MS et $\mathrm{N}$ est un indicateur de leur partage entre organes végétatifs et reproducteurs sur l'ensemble du cycle, prenant en compte les répartitions qui se sont faites au cours de la mise en place des graines et les accumulations et redistributions qui sont intervenues pendant leur remplissage. Pour $\mathrm{N}$, l'indice de récolte a été semblable dans toutes les situations, les teneurs $\mathrm{N}$ finales des différents organes sources étant plus faibles sur les parcelles NI que sur les parcelles $\mathrm{I}$. Ce résultat indique que les possibilités de remobilisation du $\mathrm{N}$ accumulé vers les graines sont indépendantes de son origine, minérale ou atmosphérique, alors que Warembourg et Fernandez (1985) ont suggéré pour le soja une remobilisation préférentielle de $\mathrm{N}$ fixé. En considérant l'ensemble des gousses, graines et cosses, l'effet de l'inoculation et/ou de la fertilisation sur la répartition de la MS observé à la fin floraison est encore perceptible à la récolte mais atténué. $L$ 'indice de récolte pour la MS, qui en revanche ne tient compte que du poids des graines, a été significativement supérieur après inoculation; ce fait 
traduit la meilleure efficacité de la MS produite visà-vis de l'élaboration du rendement quand le niveau de $\mathrm{N}$ dans la plante est élevé (Duthion, 1987). L'apport d'engrais n'a pas modifié l'indice de récolte pour la MS sur les parcelles I; la diminution de l'indice, qu'il a entraîné sur les parcelles NI, est à rapprocher des observations faites sur des espèces non fixatrices (Donald et Hamblin, 1976).

\section{Les 2 voies de la nutrition azotée}

Cultivé sur solution nutritive, le lupin blanc est apte à s'alimenter totalement à partir de $\mathrm{N}$ nitrique (Pate et al., 1979). Les résultats obtenus au champ pour des plantes non fixatrices seraient donc liés à un décalage entre leurs besoins et l'offre du milieu, décalage dans le temps, puisque l'accumulation de $\mathrm{N}$ ne devient importante qu'au début floraison plus de 2 mois après l'apport, et le décalage entre quantités offertes et quantités nécessaires à une accumulation optimale. Le mode de nutrition n'a pas en lui-même influé sur l'accumulation de $\mathrm{N}$; avec une alimentation à prédominance minérale, un ralentissement de celle-ci est intervenu au moment, d'autant plus précoce que la quantité d'engrais apporté a été faible, où la disponibilité de $\mathrm{N}$ dans le sol a diminué (Fig. 1). Les résultats de 1983, qui donnent l'application unique de $160 \mathrm{~kg} / \mathrm{ha}$ au semis pour fumure optimale, sont en contradiction partielle avec cette proposition.

Globalement, la fertilisation a réduit la part de la fixation symbiotique dans l'accumulation de $\mathrm{N}$ (Fig. 4); avec $160 \mathrm{~kg}$ appliqués au semis, elle passe de 80 à $50 \%$ pour une même quantité totale accumulée. Cette réduction apparaît relativement faible, compte tenu de beaucoup de résultats rapportés, pour le soja par exemple (Weber, 1966b; Bhangoo et Albritton, 1976; Bouniols et al., 1986). Richards et Soper (1979) ont observé en revanche sur féverole une diminution à peine plus importante avec une dose de $\mathrm{N}$ beaucoup plus élevée. Considérée non plus seulement en fin de végétation mais dans son évolution, la proportion de $\mathrm{N}$ fixé dans $\mathrm{N}$ total a beaucoup augmenté, sur les parcelles I fertilisées, de la floraison A3 à la maturité (dates 2 et 3 de la Fig. 4), croissant de 38 à $50 \%$ pour 1160 . Entre ces 2 dates, dont la première a été de peu postérieure à la chute de la disponibilité de $\mathrm{N}$ dans le sol, $\mathrm{N}$ accumulé a été presque entièrement d'origine symbiotique. Auparavant, les plantes I et $\mathrm{NI}$ ayant reçu la même dose d'engrais avaient absorbé la même quantité de $\mathrm{N}$ minéral, complétée chez les plantes I par $\mathrm{N}$ d'origine atmosphérique jusqu'à couverture de leurs besoins. Les 2 voies de la nutrition azotée apparaîtraient donc plus complémentaires qu'antagonistes, la plante absorbant préférentiellement $\mathrm{N}$ minéral disponible. L'antagonisme $\mathrm{N}$ combinésymbiose ne se serait manifesté ici qu'en condition marginale, lorsque le nombre de bactéries dans le sol a été très faible; le $\mathrm{N}$ minéral a limité les possibilités de nodulation tardive par des Rhizobium indigènes sur parcelles $\mathrm{Nl}$.

\section{Bilan N du sol}

La culture des légumineuses à graines serait susceptible, dans quelques circonstances, déficit hydrique, richesse en $\mathrm{N}$ du milieu, d'entraîner un appauvrissement en $\mathrm{N}$ du sol (Bouniols et al., 1984). Le bilan $N$ dans l'essai 1981 a été estimé par la somme de $\mathrm{N}$ d'origine symbiotique retourné au sol et de la fumure, diminuée de $\mathbf{N}$ d'origine minérale exporté par la récolte; il n'a été déficitaire dans aucune des situations créées. Compte tenu des seuls organes aériens, il a été de +6 et +60 $\mathrm{kg} / \mathrm{ha}$ pour 10 et I160; avec des mobilisations racinaires de l'ordre de $10 \%$ du $\mathrm{N}$ accumulé par la plante, estimation intermédiaire entre les quantités proposées par Hocking et Pate (1978) et Farrington et al. (1977), il aurait une valeur plus vraisemblable de +31 et $+75 \mathrm{~kg} / \mathrm{ha}$. Pour NIO et $\mathrm{NI} 160$, il a été selon les 2 modes de calcul, de 0 et $+63 \mathrm{~kg} / \mathrm{ha}$ ou de +11 et $+68 \mathrm{~kg} / \mathrm{ha}$. Après les plantes utilisées comme témoins dans la détermination de $\mathrm{N}$ fixé par la méthode isotopique, le bilan a été de - 45 et - $89 \mathrm{~kg} / \mathrm{ha}$ pour avoine et blé de printemps non fertilisés.

\section{Conséquences pour la conduite de la culture}

Les résultats confirment la validité des données proposées comme références (Duthion et al., 1987), utiles dans la perspective d'un diagnostic cultural. Toute stratégie en matière d'implantation de la culture du lupin blanc devrait par ailleurs retenir quelques-uns des éléments développés cidessus. En l'absence de facteurs défavorables, la fixation symbiotique de $\mathrm{N}$ permet à la plante d'assurer à l'optimum sa croissance, ses mobilisations en $\mathrm{N}$, le rendement en graines et protéines. La préoccupation de porter l'activité fixatrice à son niveau maximal et de l'y maintenir aussi longtemps que possible doit intervenir dans le choix des sites d'implantation et des interventions culturales. Lorsqu'elles sont toutes deux accessibles, 
les voies de la nutrition azotée paraissent complémentaires dans la couverture des besoins de la plante; cependant permettre à celle-ci d'extérioriser ses capacités de fixation est sans doute judicieux et doit influer sur sa place dans les rotations. $\mathrm{Si}$, pourtant, la culture est établie en milieu riche en $\mathrm{N}$, l'inoculation des semences est certainement nécessaire, même en présence d'un nombre jugé convenable de $R$. lupini dans le sol, pour assurer une nodulation suffisamment précoce. Enfin, corriger, en cours de végétation, une déficience de la fourniture en $\mathrm{N}$ apparaît malaisé.

\section{Références}

Amarger N. \& Duthion C. (1983) Effets de l'inoculation par Rhizobium et de la fertilisation azotée sur les rendements et la teneur en azote des graines de lupin (Lupinus albus, L. mutabilis, L. luteus). Agronomie 3, 995-1000

Bhangoo M.S. \& Albritton D.J. (1976) Nodulating and non-nodulating Lee soybean isolines response to applied nitrogen. Agron. J. 68, 642-645

Bouniols A., Puech J., Marty J.R., Hilaire A. \& Mondies M. (1984) Variabilité de la fixation symbiotique chez les légumineuses à graines dans différentes rotations culturales. Journée nationale sur les Protéagineux. In : Pourquoi Pois... ? ITCF, Paris, pp. 129-130

Bouniols A., Chalamet A., Lagacherie B., Merrien A. \& Obaton M. (1986) Nutrition azotée du soja : limites et améliorations de la fixation symbiotique. In : Le Soja, Physiologie de la plante et Adaptation aux Conditions Françaises. CETIOM, Paris, pp. 157-165

Brevedan R.E., Egli D.B. \& Leggett J.E. (1978) Influence of $N$ nutrition on flower and pod abortion and yield of soybeans. Agron. J. 70, 81-84

Donald C.M. \& Hamblin J. (1976) The biological yield and harvest index of cereals as agronomic and plant breeding criteria. Adv. Agron. 28, 361-405

Duthion C. (1987) Accumulation d'azote et formation du rendement chez le lupin blanc (Lupinus albus L.), In : Nutrition Azotée des Légumineuses (P. Guy, ed.), INRA, Paris, pp. 293-298

Duthion C., Amarger N. \& Mariotti A. (1987) Accurnulation potentielle de matière sèche et d'azote chez le lupin blanc de printemps (Lupinus albus L.). Agronornie 7, 585-593

Farrington P., Greenwood E.A.N., Titmanis Z.V., Tririck
M.J. \& Smith D.W. (1977) Fixation, accumulation and distribution of nitrogen in a crop of Lupinus angustifolius cv Unicrop. Aust. J. Agric. Res. 28, 237-248

Hanway J.J. \& Weber C.R. (1971 a) Dry matter accumulation in soybean (Glycine max (L.) Merril) plants as influenced by N, P and K fertilization. Agron. J. 63, 263266

Hanway J.J. \& Weber C.R. (1971 b) Accumulation of N, $\mathrm{P}$ and $\mathrm{K}$ by soybean (Glycine max (L.) Merrill) plants Agron. J. 63, 406-408

Hill G.D. (1977) The composition and nutritive value of lupin seed. Nutr. Abstr. Rev. B, 47, 511-529

Hocking P.J. \& Pate J.S. (1978) Accumulation and distribution of mineral elements in annual lupins Lupinus albus L. and Lupinus angustifolius L. Aust. J. Agric. Res. 29, 267-280

Merbach W., Stelzner C. \& Stock H. G. (1980) Influence of nitrogen fertilization on yield and seed protein composition of white lupins in field trials. Arch. Acker. Pflanzenbau Bodenkd. 24, 47-53 (In : Soils Fertil., 1980, 10563)

Monfort B. (1978) Variation de la quantité d'azote fixé symbiotiquement par un lupin blanc cultivé en serre en présence d'azote minéral. Mémoire ENITA, DijonQuétigny

Obaton M., Miquel M., Robin P., Conejero G., Domenach A.M. \& Bardin R. (1982) Influence du déficit hydrique sur l'activité nitrate-réductase et nitrogénase chez le soja. C.R. Acad. Sci. Paris 294, 1007-1012

Pal U.R. \& Saxena M.C. (1976) Relationships between nitrogen analysis of soybean tissues and soybean yields. Agron. J. 68, 927-932

Pate J.S., Layzell D.G. \& Atkins C.A. (1979) Economy of carbon and nitrogen in a nodulated and non nodulated ( $\mathrm{NO}_{3}$-grown) legume. Plant Physiol. 64, 1083-1088

Penning de Vries F.W.T. (1982) Crop production in relation to availability of nitrogen. $I n$ : Simulation of Plant Growth and Crop Production (F.W.T. Penning de Vries \& H.H. Van Laar, eds), Pudoc, Wageningen, pp. 213-221

Richards J.E. \& Soper R.J. (1979) Effect of N fertilizer on yield, protein content and symbiotic $\mathrm{N}$ fixation in fababeans. Agron. J. 71, 807-811

Warembourg F.R. \& Fernandez M.P. (1985) Distribution and remobilization of symbiotically fixed nitrogen in soybean (Glycine max). Physiol. Plant 65, 281-286

Weber C.R. (1966 a) Nodulating and non nodulating soybean isolines. I. Agronomic and chemical attributes. Agron. J. 58, 43-46

Weber C.R. (1966 b) Nodulating and non nodulating soybean isolines. II. Response to applied nitrogen and modified soil conditions. Agron. J. 58, 46-49 